\title{
Prevalence of dyspepsia in patients with cholecystolithiasis: a systematic review and meta-analysis
}

Carmen S.S. Latenstein ${ }^{\mathrm{a}, *}$, Judith J. de Jong ${ }^{\mathrm{b}, *}$, Jedda J. Eppink ${ }^{\mathrm{b}}$, Marten A. Lantinga ${ }^{\mathrm{a}}$, Cornelis J.H.M. van Laarhoven ${ }^{a}$, Philip R. de Reuver ${ }^{a}$ and Joost P.H. Drenth ${ }^{b}$

\begin{abstract}
Cholecystolithiasis and functional gastrointestinal disorders are both highly prevalent in the industrialized world and may exist concomitantly. The presence of both conditions impedes identification of the source of symptoms, leading to a risk of ineffective cholecystectomies with lack of symptom resolution. We carried out a systematic review and meta-analysis to determine the prevalence of dyspepsia in patients with uncomplicated cholecystolithiasis. The electronic databases Medline, Embase, and Web of Science were searched for articles reporting the prevalence of dyspepsia in adults ( $\geq 18$ years) with uncomplicated cholecystolithiasis. Pooled prevalence and $95 \%$ confidence interval were calculated. $I^{2}$ statistics were used to determine heterogeneity and the Methodological Evaluation of Observational Research criteria were applied for quality assessment. The study was reported according to the Preferred Reporting Items for Systematic Reviews and Meta-Analyses statement. Of the 1696 studies evaluated, 13 reported the prevalence of dyspepsia in a total of 1227 cholecystolithiasis patients seeking medical care. The pooled prevalence of dyspepsia in patients with cholecystolithiasis was 65.7\% (95\% confidence interval: 51-79\%). However, heterogeneity was large across studies. Overall, three studies used validated diagnostic criteria. Variation in diagnostic measures significantly influenced the prevalence of dyspepsia. In conclusion, symptoms similar to those of functional gastrointestinal disorders are common in patients with cholecystolithiasis, obscuring the source of abdominal complaints. Tools to select patients who will benefit from cholecystectomy are paramount to prevent ineffective surgery. Eur $\mathrm{J}$ Gastroenterol Hepatol 31:928-934

Copyright @ 2019 The Author(s). Published by Wolters Kluwer Health, Inc.
\end{abstract}

\section{Introduction}

Gallstone disease is a significant burden in the industrialized world, in particular among middle-aged women, as point estimates indicate a prevalence of $5-22 \%[1,2]$. Cholecystectomy is now the most common elective abdominal surgery performed in the USA, with over 750000 operations being performed

European Journal of Gastroenterology \& Hepatology 2019, 31:928-934

Keywords: cholecystectomy, cholecystolithiasis, dyspepsia, epidemiology, functional gastrointestinal disorders, meta-analysis

Departments of a Surgery and ${ }^{b}$ Gastroenterology, Radboud University Medical Centre, Nijmegen, The Netherlands

Correspondence to Carmen S.S. Latenstein, BSc, Department of Surgery, Radboud University Medical Center, PO Box 9101, 6500 HB Nijmegen, The Netherlands

Tel: + 31243613 983; fax: +31243635 115;

e-mail: carmen.latenstein@radboudumc.nl

${ }^{\star}$ Carmen S.S. Latenstein and Judith J. de Jong contributed equally to the writing of this article.

This research was presented at the Digestive Disease Days. Veldhoven, The Netherlands on 5 October 2018.

Received 5 March 2019 Accepted 26 April 2019

Supplemental Digital Content is available for this article. Direct URL citations appear in the printed text and are provided in the HTML and PDF versions of this article on the journal's website, www.eurojgh.com.

This is an open-access article distributed under the terms of the Creative Commons Attribution-Non Commercial-No Derivatives License 4.0 (CCBY-NC$\mathrm{ND}$ ), where it is permissible to download and share the work provided it is properly cited. The work cannot be changed in any way or used commercially without permission from the journal. annually [3], although an element of overuse undoubtedly exists. Similarly, the global prevalence of functional gastrointestinal disorders (FGID), particularly functional dyspepsia, is high $(20 \%$ [4]). Again, in particular, middle-aged women are at risk of developing functional dyspepsia.

FGID encompasses a group of multifactorial disorders. Disturbances of the bidirectional brain-gut interaction are recognized in the pathophysiology of FGID, as well as visceral hypersensitivity and impaired gastrointestinal motility [5]. Symptoms roughly correlate with the level of the gastrointestinal tract that is affected. FGID that involves the upper or the mid gastrointestinal tract (i.e. functional dyspepsia) is associated with pain or discomfort located in the upper abdomen.

Although asymptomatic in the majority of patients, symptomatic cholecystolithiasis may typically present as biliary colic [6]. Patients may experience abdominal pain in the upper right quadrant, frequently radiating to the back, lasting over $30 \mathrm{~min}$, and often accompanied by nausea or vomiting. However, in many cases, a broad range of atypical symptoms, such as postprandial pain and bloating, accompany biliary colic or even dominate presenting symptoms [7].

Several studies show that $\sim 40 \%$ of patients do not gain relief from abdominal pain despite cholecystectomy [8-10], suggesting that postoperative symptoms must be attributed to a different source. Functional dyspepsia may be overlooked in patients reporting abdominal symptoms with cholecystolithiasis and could explain a lack of symptom 
resolution. Currently, it is unclear to what extent FGID plays a role in these patients.

In view of the varying prevalence of FGID in patients with cholecystolithiasis and potential impact on the choice of treatment, there is a need for unambiguous data. We carried out a systematic review and meta-analysis of literature to determine the prevalence of dyspepsia in a healthcare-seeking population with symptomatic, uncomplicated cholecystolithiasis. General practitioners, gastroenterologists, and surgeons could use these results in future decision-making.

\section{Methods}

This study was reported according to the Preferred Reporting Items for Systematic Reviews and Meta-Analyses (PRISMA) statement [11]. Details of the study protocol were registered on PROSPERO [12]. For this study, IRB approval and informed consent were not needed.

\section{Search strategy and study selection}

A systematic search of the literature was performed in November 2017 using electronic databases Medline (1946-November 2017), Embase (1974-November 2017), and Web of Science (1945-November 2017).

Articles eligible for inclusion had study populations that included adults of at least 18 years with cholecystolithiasis, who consulted healthcare services, and in whom the prevalence of dyspepsia was assessed. In the case of a surgical clinic-based setting, only preoperative assessments were considered for inclusion. Cholecystolithiasis was defined as the presence of one or more stones in the gallbladder. Uncomplicated cholecystolithiasis was defined as gallstones in the gallbladder, in the absence of stones in bile ducts, cholecystitis, or cholangitis. The combination of gallstones and any abdominal symptom was indicated as symptomatic cholecystolithiasis.

Only studies published after the introduction of laparoscopic cholecystectomy in 1985 were included, to represent current clinical practice. We limited our search to prospective and cross-sectional studies. Abstracts without published full texts were included if all data for primary and secondary endpoints were available. Studies solely studying gallbladder dyskinesia and biliary dyspepsia were excluded. In addition, studies with complicated gallstone disease patients were excluded in case no distinction was made between complicated and uncomplicated disease. Complicated gallstone disease was defined as the presence of acute cholecystitis, choledocholithiasis, cholangitis, and biliary pancreatitis. All eligibility criteria are shown in Supplementary Table S1 (Supplemental digital content 1, http://links.lww.com/EJGH/ A418 in the supplementary files).

To build a search, the following terms were used for FGID: abdominal pain, functional gastrointestinal disorders, FGID, dyspepsia, and indigestion. These terms were combined with the set operator ' $O R$ '. The terms for FGID were then combined using the set operator 'AND' with studies identified with the terms gallstone, biliary calculi, cholecystolithiasis, cholelithiasis, and gallstones. Details of the search are shown in the Supplementary Table S2 (Supplemental digital content 2, http://links.lww. com/EJGH/A419). In addition, for every keyword
[Medical Subject Headings (MeSH) for Medline and Emtree for Embase], free text words were searched. The Cochrane Library was checked with identical keywords. Cross-references were hand-searched. Two reviewers (J.E. and C.L.) independently screened titles and abstracts. A third reviewer (J.J.) resolved differences in article selection and verified selected articles by means of discussion.

\section{Data extraction}

A predefined data extraction form was used. Data were extracted independently by two reviewers (J.E. and C.L.), and discrepancies were resolved by the third reviewer (J.J). The following study characteristics were extracted: first author, year of publication, country, study design, patient inclusion and exclusion criteria, type of gastrointestinal symptoms studied, questionnaire or criteria to define the functional symptoms, and sample size. The following additional data were collected for each study: mean age of patients, sex, and prevalence of dyspepsia. Authors were contacted in case of missing data. Discrepancies in data extraction were discussed until a consensus was reached.

\section{Quality assessment}

The methodological quality of the studies included was assessed independently by two reviewers (J.E. and C.L.) using the Methodological Evaluation of Observational Research (MORE) [13] and discrepancies were resolved by the third reviewer (J.J.). This quality assessment tool tests components of the internal and external validity and reporting of descriptives of the studies and creates a validity quality report for each study.

\section{Data synthesis}

The proportion of patients with dyspepsia was defined as the number of patients with a diagnosis of dyspepsia before treatment for cholecystolithiasis divided by the total number of patients with uncomplicated cholecystolithiasis. The term 'dyspepsia' was pragmatically used in this review as a description of upper gastrointestinal complaints, notwithstanding that the Rome criteria require measures to exclude organic disorders for functional dyspepsia [14]. The proportions of dyspepsia were combined to calculate the pooled prevalence of dyspepsia. Methods of measurement were divided into three categories: validated questionnaires, single symptom, broad definition, and undefined dyspepsia. The broad definition was defined according to the 1988 Working Party report, which defined dyspepsia as upper abdominal or retrosternal pain, discomfort, heartburn, nausea, vomiting, or other symptoms considered to be referable to the proximal alimentary tract [15].

\section{Statistical analysis}

A random-effects model was used for the pooled estimates and $95 \%$ confidence intervals (CIs) as this would yield the most conservative estimate. The random-effects model was constructed using MedCalc statistical software version 18.2.1 (MedCalc Software bvba, Ostend, Belgium; 2018). Continuous data are reported as mean with SD. We used $I^{2}$ statistics to determine heterogeneity for pooled estimates. 
If appropriate, publication bias will be assessed using a funnel plot, Begg's rank correlation method, and Egger's regression method.

\section{Results}

\section{Selected studies}

The search strategy identified 1696 articles. After excluding duplicates and selection on the basis of title and abstract, 63 articles were screened on full text, of which finally 13 were included in this review (Fig. 1). Screening references and contacting study authors did not yield additional studies.

\section{Characteristics}

Study characteristics are summarized in Table 1. A total of 1227 patients from 13 studies were included. The majority of studies were prospective, but in two a cross-sectional design, was used $[16,17]$. Three studies used a validated questionnaire [18-20]. Four studies used a 'broad definition [21-24], all to define dyspepsia. In two studies, a single symptom out of a list

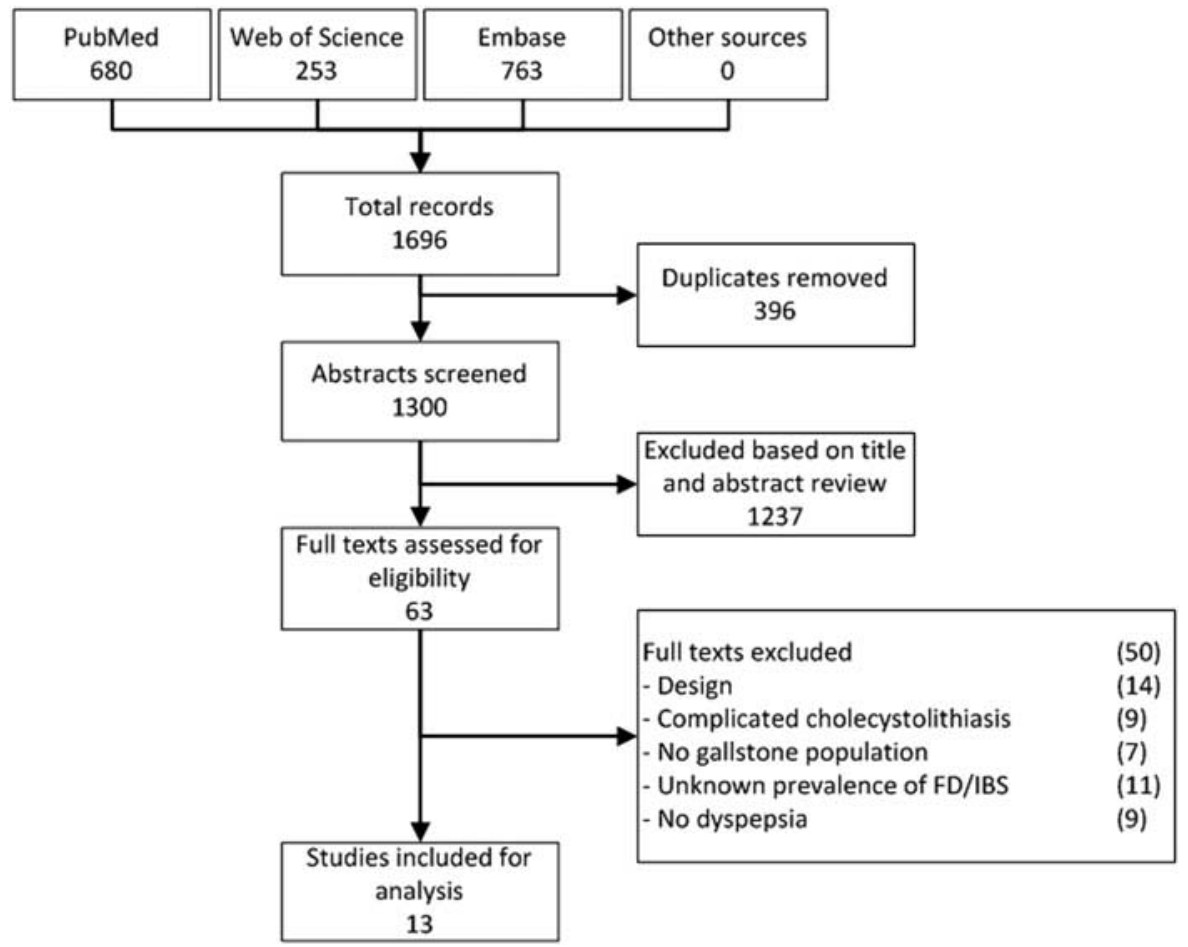

Fig. 1. Study identification and selection flow chart. Flow chart of study selection using three databases and cross-referencing. FD, functional dyspepsia; IBS, irritable bowel syndrome.

Table 1. Study characteristics

\begin{tabular}{|c|c|c|c|c|c|c|}
\hline References & Study design & $\begin{array}{l}\text { Method of } \\
\text { measurement }\end{array}$ & Sample size & $\begin{array}{c}\text { Age (range or mean } \pm \mathrm{SD} \text { ) } \\
\text { (years) }\end{array}$ & Male (\%) & $\begin{array}{l}\text { Patients with gallstones and } \\
\text { gastrointestinal symptoms (\%) }\end{array}$ \\
\hline Lorusso et al. [19] & Single-center prospective & GSRS $^{c}$ & 124 & $44.4 \pm 12^{a}$ & 19.2 & 46.7 \\
\hline Mertens et al. [21] & Single-center prospective & Broad definition & 183 & $46.0 \pm 11$ & 25.7 & 66.7 \\
\hline Ibrarullah et al. [22] & Prospective & Broad definition & 43 & $36.8(19-62)$ & 23.3 & 67.4 \\
\hline Schmidt et al. [18] & Single-center prospective & Simplified Rome II & 153 & $48(17-85)$ & 20.3 & 87.6 \\
\hline Portincasa et al. [16] & Cross-sectional & Single symptom & 20 & $44(21-67)$ & 35.0 & 60.0 \\
\hline Borly et al. [23] & Single-center prospective & Broad definition & 80 & $44(20-70)$ & 11.2 & 86.0 \\
\hline Berhane et al. [17] & Single-center cross-sectional & Single symptom & 166 & $47(19-79)^{\mathrm{b}}$ & 26.0 & 98.0 \\
\hline Berger et al. [20] & Multicenter prospective & $\begin{array}{c}\text { Self-administered } \\
\text { symptom } \\
\text { questionnaire }^{c}\end{array}$ & 107 & $45.7(17-79)$ & 19.6 & 82.7 \\
\hline Bagaria et al. [25] & Single-center prospective & Undefined criteria & 25 & 42.1 & 20.0 & 40 \\
\hline Karmacharya et al. [26] & Single-center prospective & Undefined criteria & 96 & $40.3(20-75)$ & 12.5 & 33.3 \\
\hline Kumar et al. [27] & Single-center Prospective & Undefined criteria & 100 & 43.7 & 20.0 & 40 \\
\hline Misrani et al. [28] & Single-center cross-sectional & Undefined criteria & 100 & $44(20-70)$ & 20.0 & 50 \\
\hline Abu Farsakh et al. [24] & Single-center prospective & Broad definition & 30 & $43.8(22-70)$ & 16.7 & 66.7 \\
\hline
\end{tabular}

Questionnaire validated by authors through previous research.

GSRS, Gastrointestinal Symptom Rating Scale.

Broad definition, a group of gastrointestinal symptoms selected by the authors, which together form the definition dyspepsia; Single-symptom definition, one gastrointestinal suffices to diagnose dyspepsia; Undefined, symptoms are classified as 'dyspepsia', but no criteria for the diagnosis were presented.

${ }^{a}$ Mean age at the time of follow-up.

blncluding complicated gallstone disease.

${ }^{\mathrm{C}}$ Questionnaire validated by authors through previous research. Besides, this applies to the 'Simplified Rome II' questionnaire of Schmidt et al. [18] as well. 
of symptoms ('single-symptom') was sufficient to diagnose dyspepsia. Four studies did not define the definition used for dyspepsia (undefined) [25-28]. The low number of studies and high heterogeneity did not allow for a funnel plot or regression-based assessments for publication bias.

\section{Quality Assessment}

The quality assessment of the 14 articles was performed using the MORE quality assessment. Figure 2 presents the internal and external validity of all studies and reporting of descriptives (full quality assessment is presented in Supplementary Table S3, Supplemental digital content 3, http://links.lww.com/EJGH/A420 and Supplementary Table S4, Supplemental digital content 4, http://links.lww.com/ EJGH/A421). Regarding to internal validity, all except three studies had a major flaw, mostly because they did not assess the severity of disease. In terms of external validity, all studies had a major flaw because of a hospital-based setting. As this was an inclusion criterion for our study, the limited relevance should be kept in mind during interpretation. In terms of reporting of descriptives, six studies did not report ethical approval of their study, 11 did not declare conflicts of interest, and eight did not report funding of the study. It should be noted that dyspepsia was a secondary outcome in most studies and was therefore likely to be analyzed less extensively.

\section{Prevalence of dyspepsia}

The prevalence of dyspepsia in patients with symptomatic uncomplicated cholecystolithiasis ranged from 33 to $98 \%$. The study that presented the lowest dyspepsia prevalence was from Nepal and used undefined criteria [26].

The pooled prevalence for dyspepsia across all 13 studies was $65.7 \%$ (95\% CI: 51.1-79.0), with heterogeneity between (a)

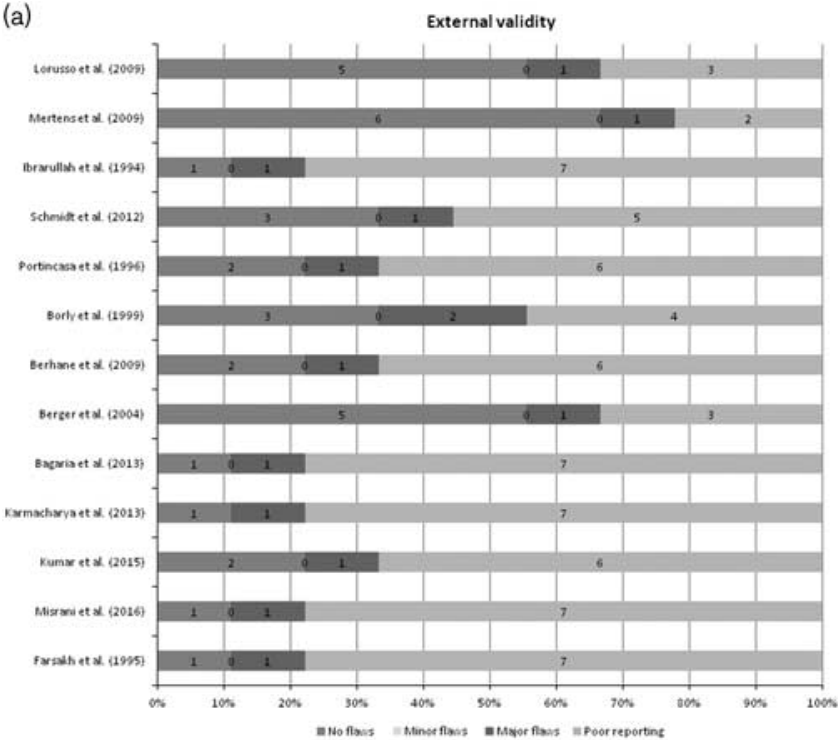

(b)

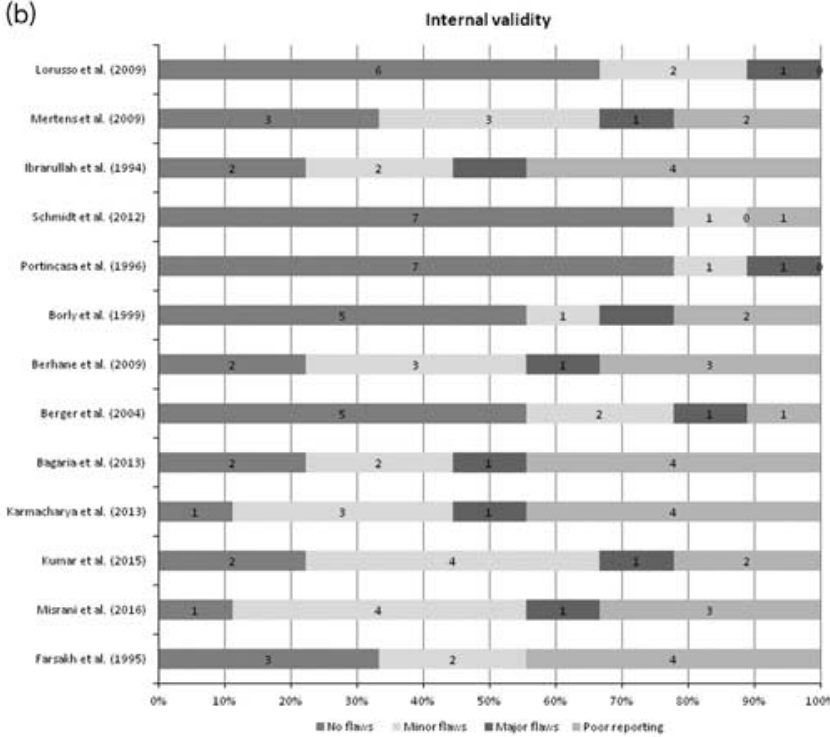

(c)

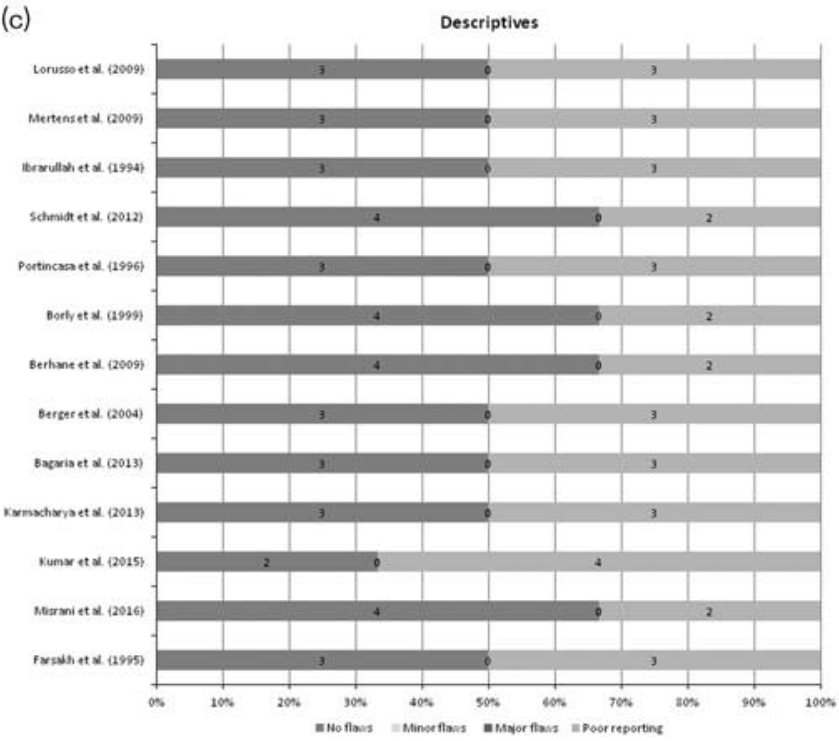

Fig. 2. Quality assessment. (a) Quality assessment external validity. (b) Quality assessment internal validity. (c) Quality assessment descriptives. Assessment of internal and external validity and reporting of descriptive of the studies included according to the Methodological Evaluation of Observational Research (MORE) criteria [31], modified to fit our target studies. Major and minor flaws were pre-specified separately for internal and external validity and reporting of descriptives. Reporting quality was scored using the option 'poor reporting' for all quality criteria. 


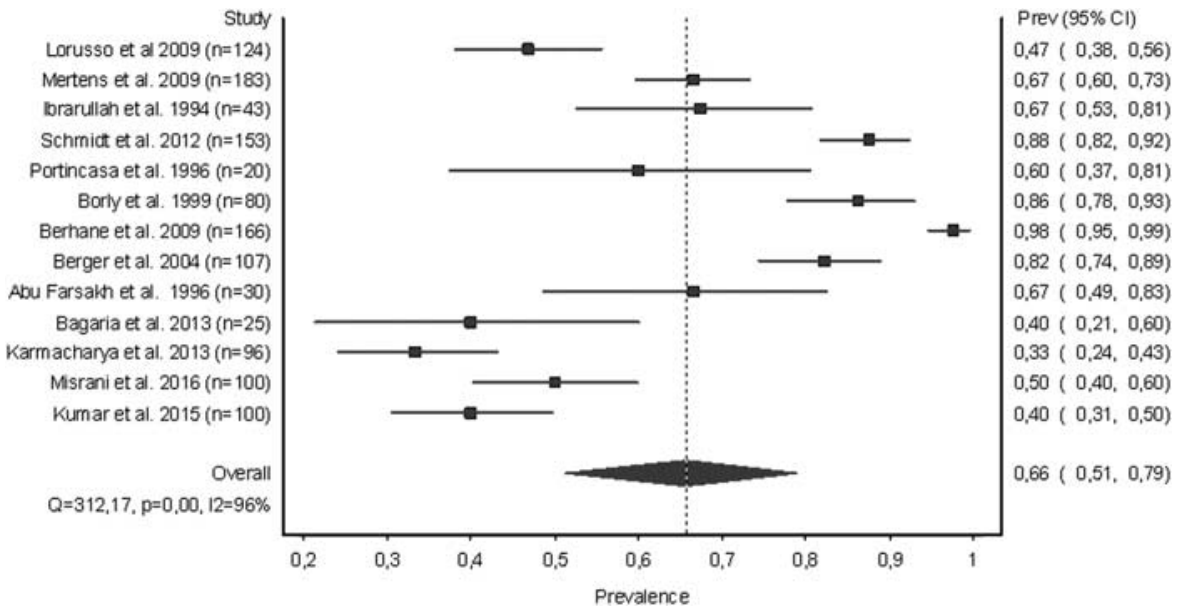

Fig. 3. Forrest plot (random effects). Forrest plot of meta-analysis on the prevalence of dyspepsia in patients with symptomatic uncomplicated cholecystolithiasis. Squares indicate the prevalence of separate studies; the horizontal line indicates 95\% confidence interval (Cl); and the diamond indicates the summary risk estimate with its $95 \% \mathrm{Cl}$.

Table 2. Pooled prevalence of dyspepsia and subgroup analysis by diagnostic tool

\begin{tabular}{lcccc}
\hline & $\begin{array}{c}\text { Number of } \\
\text { studies }\end{array}$ & $\begin{array}{c}\text { Number of } \\
\text { patients }\end{array}$ & $\begin{array}{c}\text { Pooled prevalence } \\
{[\%(95 \%} \\
\text { confidence interval) }]\end{array}$ & $I^{2}[\%(P)]$ \\
\hline $\begin{array}{c}\text { Pooled prevalence } \\
\text { Dyspepsia }\end{array}$ & 13 & 1.227 & $65.7(51.1-79.0)$ & $96(<0.01)$ \\
$\begin{array}{c}\text { Pooled prevalence by } \\
\text { diagnostic tool }\end{array}$ & 3 & 384 & $75.4(46.4-96.3)$ & $97(<0.01)$ \\
$\begin{array}{c}\text { Validated } \\
\text { questionnaire }\end{array}$ & 4 & 336 & $72.6(60.8-83.1)$ & $77(<0.01)$ \\
$\begin{array}{c}\text { Broad definition } \\
\text { Single-symptom } \\
\text { definition }\end{array}$ & 2 & 186 & $80.8(37.5-100)$ & $93(<0.01)$ \\
$\begin{array}{c}\text { Undefined } \\
\text { dyspepsia }\end{array}$ & 4 & 321 & $41.0(33.4-48.8)$ & $47(0.13)$ \\
& & & &
\end{tabular}

Pooled prevalence generated through a random-effects model. Heterogeneity between studies is represented by $l^{2}$.

Broad definition, a group of gastrointestinal symptoms selected by the authors, which together form the definition of dyspepsia; Single-symptom definition, one gastrointestinal suffices to diagnose dyspepsia; Undefined, symptoms are classified as 'dyspepsia', but no criteria for the diagnosis were presented.

studies $\left(I^{2}=96 \%, P<001\right)$, as shown in Fig. 3. Subgroup analysis of prevalence by method of symptom measurement showed pooled prevalence's of $75.4 \%$ (95\% CI: 46.4-96.3), $72.6 \%$ (95\% CI: 60.8-83.1), 80.8 (95\% CI: 37.5-100), and 41.0 (95\% CI: 33.4-48.8) for validated questionnaires, broad definition, single symptom, and undefined, respectively. The subgroup prevalences in the broad definition group were not statistically different from the overall prevalence, while $P$ was less than 0.01 for the validated questionnaires, single symptom, and undefined group. The pooled prevalence of the total and subgroup analyses is shown in Table 2.

\section{Discussion}

Sixty percent of patients with uncomplicated cholecystolithiasis have gastrointestinal symptoms that share features with functional dyspepsia, which is higher compared with the general population [4,29]. We found use of a broad variety of diagnostic tools across studies to diagnose dyspepsia, only three of which were validated.
To put our data into perspective, in the UK, over 50000 cholecystectomies are performed yearly, mostly for symptomatic cholecystolithiasis. According to our data, around 33000 of these patients will have both cholecystolithiasis and dyspepsia [30]. Assumedly, many patients are subjected to cholecystectomy because of dyspeptic symptoms rather than symptomatic gallstones, subsequently causing long-term persistent abdominal complaints. Three large studies showed similar proportions of persistent symptoms, viz. 37 [31], 40.4 [19], and 59.5\% [32]. Previous literature suggests that if the preoperative assessment is standardized and other possible causes of abdominal symptoms are ruled out, the postoperative symptomatic outcome is significantly improved [33,34]. These symptoms are a significant burden on patients and healthcare as $56 \%$ of patients will need additional healthcare, against median costs of $\$ 555$ per year per patient. Sick leave and production loss of employed patients add an additional $\$ 361$ per year per patient for work-related costs [35].

A lower prevalence of dyspeptic symptoms is seen in population-based studies with 'healthy', unselected patients $[36,37]$. Previously, the variation between hospital-based and population-based prevalences of dyspeptic symptoms was confirmed and no causal relationship was found between dyspeptic symptoms and gallstones [38,39]. Only biliary colic, radiating pain, and use of analgesics were related to gallstones, supporting our hypothesis that dyspeptic symptoms may not be mainly caused by gallstones. Based on these results, cholecystectomy for dyspeptic symptoms alone, which has been common practice in the past, is unjustified and emphasizes the need for stringent indications for cholecystectomy.

The majority of the studies included were European or South-Asian. Intercontinental differences in the general prevalence of dyspepsia and cholecystolithiasis prevent extrapolation of our results to other continents [4]. However, as cholecystolithiasis - of the cholesterol gallstone type - and FGID are predominantly western diseases, this review was in advance expected to mainly impact western countries $[4,40]$. Geographical differences did not cause heterogeneity between studies. 
We included studies providing the prevalence of gastrointestinal symptoms were labeled 'dyspepsia', to increase homogeneity. First, authors' interpretation of gastrointestinal symptoms being dyspepsia was used to define our primary outcome. As validated questionnaires or additional investigations were rarely used, our presented prevalences are an estimation. Second, the number of eligible articles was affected. As sample sizes were low and a variety of diagnostic tools were used, we found considerable statistical heterogeneity between studies and wide CIs. The risk of small-study effects should be kept in mind during interpretation of the results as the actual prevalence may be overestimated. Still, data generated in this study provide an important directional vision on appropriate management of abdominal pain and further research into this topic should be carried out.

The absence of desired variables is a drawback often encountered in meta-analyses. In our case, insufficient information was available on indications for cholecystectomy, laboratory values, and consistent information on persistence of symptoms. In addition, the general quality of the studies was limited, mainly because of poor reporting, low sample sizes, and lack of use of validated instruments. Extracted prevalences were most often secondary outcomes of the studies included, for which consequently studies were not powered. Strengthening our study is the use of the PRISMA statement throughout this review to ensure a robust methodology and sufficient quality [11,41]. The MORE criteria were used to assess the reporting quality of the articles included [13]. Furthermore, cross-referencing was performed and authors of incomplete data or missing full-texts were contacted for optimal yield of our search. The result of our search provides an important overview of the current literature and highlights the lacunas yet to be filled.

Whereas symptomatic cholecystolithiasis is an indication for cholecystectomy, the asymptomatic presence of gallstones is not as only $3-5 \%$ progress to complicated cholecystolithiasis [42]. To attribute abdominal symptoms to FGID, in the presence of gallstones, diagnostic criteria are required. Dyspepsia is a multifactorial disorder that remains difficult to define. Important progress in the Rome criteria was made with the newly presented clusters 'postprandial distress syndrome' and 'Epigastric Pain Syndrome' [43]. In addition, the current pathophysiologically driven definition of dyspepsia contributes to the distinction between disorders, such as dyspepsia and symptomatic cholecystolithiasis [14]. As soon as diagnostic criteria for FGID in patients with gallstones are sufficiently clarified, algorithms may be promising future clinical tools to aid decision-making. Especially when digitalized and individually adjustable, as with many virtual assessors, such contemporary measures have been shown to effectively select appropriate clinical pathways [44].

Cholecystolithiasis and FGIDs are both associated with female sex and lifestyle factors. Fatty foods are a well-known trigger of symptoms in functional dyspepsia [45] as well as of biliary colic [6]. Targeting the shared lifestyle factor or initiating other treatments for dyspepsia therefore seems appropriate in a comorbid population. An initial conservative approach may provide exciting opportunities for future reduction of ineffective cholecystectomies. This notwithstanding, satisfactory treatment of FGID is a challenge. Often, patients and clinicians conveniently choose to focus treatment on radiologically confirmed abnormalities rather than adopt the complicated path of treating FGID.

Currently, several studies are being carried out by our group in the field of appropriate cholecystectomies and persistent symptoms. One of these studies is a Dutch nationwide randomized-controlled trial in which persistent symptoms and practice variation are compared between the usual and a restrictive strategy in patients with symptomatic cholecystolithiasis [34]. In a multicenter observational study (Dutch Trial Register 2018: NTR7307, http://www.trialregister.nl/ trialreg/admin/rctview.asp?TC=7307), FGID according to the Rome IV criteria are measured in gallstone patients before and after surgery to identify FGID as a possible cause of persistent symptoms.

\section{Conclusion}

A majority of patients with cholecystolithiasis have symptoms similar to functional dyspepsia. Physicians who encounter patients with gallstones and nonspecific abdominal symptoms should be aware of this phenomenon. Measures are needed to select patients who will benefit from cholecystectomy to prevent inappropriate surgery.

\section{Acknowledgements}

Carmen S.S. Latenstein, Judith J. de Jong, and Jedda J. Eppink contributed substantially to the conception and design of the work, contributed to acquisition, analysis, and interpretation of data for the work, drafted the manuscript, approved the final version to be published, and agreed to be accountable for all aspects of the work in ensuring that questions related to the accuracy or integrity of any part of the work are appropriately investigated and resolved.

Jedda J. Eppink, Marten A. Lantinga, Cornelis J.H.M. van Laarhoven, Philip R. de Reuver, and Joost P.H. Drenth interpreted the data for the work, revised the manuscript critically for important intellectual content, approved the final version to be published, and agree to be accountable for all aspects of the work in ensuring that questions related to the accuracy or integrity of any part of the work are appropriately investigated and resolved.

\section{Conflicts of interest}

There are no conflicts of interest.

\section{References}

1 Shaffer EA. Gallstone disease: epidemiology of gallbladder stone disease. Best Pract Res Clin Gastroenterol 2006; 20:981-996.

2 Everhart JE, Khare M, Hill M, Maurer KR. Prevalence and ethnic differences in gallbladder disease in the United States. Gastroenterology 1999; 117:632-639.

3 Russo MW, Wei JT, Thiny MT, Gangarosa LM, Brown A, Ringel Y, et al. Digestive and liver diseases statistics, 2004. Gastroenterology 2004; 126:1448-1453.

4 Ford AC, Marwaha A, Sood R, Moayyedi P. Global prevalence of, and risk factors for, uninvestigated dyspepsia: a meta-analysis. Gut 2015; 64:1049-1057.

5 Jung HK, Talley NJ. Role of the duodenum in the pathogenesis of functional dyspepsia: a paradigm shift. J Neurogastroenterol Motil 2018; 24:345-354.

6 Portincasa P, Moschetta A, Petruzzelli M, Palasciano G, Di Ciaula A, Pezzolla A. Gallstone disease: symptoms and diagnosis of gallbladder stones. Best Pract Res Clin Gastroenterol 2006; 20:1017-1029. 
7 Diehl AK. Symptoms of gallstone disease. Baillieres Clin Gastroenterol 1992; 6:635-657.

8 Lamberts MP, Lugtenberg M, Rovers MM, Roukema AJ, Drenth JP, Westert GP, et al. Persistent and de novo symptoms after cholecystectomy: a systematic review of cholecystectomy effectiveness. Surg Endosc 2013; 27:709-718.

9 Thistle JL, Longstreth GF, Romero Y, Arora AS, Simonson JA, DiehI NN, et al. Factors that predict relief from upper abdominal pain after cholecystectomy. Clin Gastroenterol Hepatol 2011; 9:891-896.

10 Vetrhus M, Berhane T, Soreide O, Sondenaa K. Pain persists in many patients five years after removal of the gallbladder: observations from two randomized controlled trials of symptomatic, noncomplicated gallstone disease and acute cholecystitis. J Gastrointest Surg 2005; 9:826-831.

11 Moher D, Liberati A, Tetzlaff J, Altman DG. Preferred reporting items for systematic reviews and meta-analyses: The PRISMA Statement. PLoS Med 2009; 6:e1000097.

12 Mrak K, Eberl T, Tschmelitsch J, Langner C. Heterotopic pancreatic tissue in the cystic duct: complicating factor or coexisting pathology. South Med J 2010; 103:471-473.

13 Shamliyan T, Ansari M, Raman G, Berkman N, Grant M, Janes G, et al. Development and implementation of the standards for evaluating and reporting epidemiologic studies on chronic disease incidence or prevalence. Am J Public Health Res 2013; 1:183-190.

14 Drossman DA. Functional gastrointestinal disorders: history, pathophysiology, clinical features and Rome IV. Gastroenterology 2016. [Epub ahead of print].

15 [No authors listed]. Management of dyspepsia: report of a working party. Lancet 1988; 1:576-579.

16 Portincasa P, Di Ciaula A, Palmieri V, Velardi A, VanBerge-Henegouwen GP, Palasciano G. Impaired gallbladder and gastric motility and pathological gastro-oesophageal reflux in gallstone patients. Eur J Clin Invest 1997; 27:653-661.

17 Berhane T, Vetrhus M, Hausken T, Olafsson S, Sondenaa K. Pain attacks in non-complicated and complicated gallstone disease have a characteristic pattern and are accompanied by dyspepsia in most patients: the results of a prospective study. Scand J Gastroenterol 2006; 41:93-101.

18 Schmidt M, Sondenaa K, Dumot JA, Rosenblatt S, Hausken T, Ramnefjell M, et al. Post-cholecystectomy symptoms were caused by persistence of a functional gastrointestinal disorder. World $J$ Gastroenterol 2012; 18:1365-1372.

19 Lorusso D, Porcelli P, Pezzolla F, Lantone G, Zivoli G, Guerra V, et al. Persistent dyspepsia after laparoscopic cholecystectomy. The influence of psychological factors. Scand J Gastroenterol 2003; 38:653-658.

20 Berger MY, Olde Hartman TC, van der Velden JJ, Bohnen AM. Is biliary pain exclusively related to gallbladder stones? A controlled prospective study. Br J Gen Pract 2004; 54:574-579.

21 Mertens MC, De Vries J, Scholtes VP, Jansen P, Roukema JA. Prospective 6 weeks follow-up post-cholecystectomy: the predictive value of pre-operative symptoms. J Gastrointest Surg 2009; 13:304-311.

22 Ibrarullah M, Mittal BR, Agarwal DK, Das BK, Kaushik SP. Gastricemptying in patients with gallstone disease with or without dyspepsiaeffect of cholecystectomy. Aust N Z J Surg 1994; 64:247-250.

23 Borly L, Anderson IB, Bardram L, Christensen E, Sehested A, Kehlet H, et al. Preoperative prediction model of outcome after cholecystectomy for symptomatic gallstones. Scand J Gastroenterol 1999; 34:1144-1152.

24 Abu Farsakh NA, Stietieh M, Abu Farsakh FA. The postcholecystectomy syndrome. A role for duodenogastric reflux. J Clin Gastroenterol 1996; 22:197-201.

25 Bagaria D, Kaman L, Roger E, Dahyia D, Singh R, Bhattacharya A. Effect of laparoscopic cholecystectomy on gastric emptying and dyspepsia. Surg Endosc 2013; 27:3116-3120.
26 Karmacharya A, Malla BR, Joshi HN, Gurung RB, Rajbhandari M. The predictive value of pre-operative symptoms including upper gastrointestinal endoscopy before laparoscopic cholecystectomy for elective symptomatic cholecystolithiasis. Kathmandu Univ Med J (KUMJ) 2013; 11:300-304.

27 Kumar A, Kumar U, Munghate A, Bawa A. Role of routine intraoperative cholangiography during laparoscopic cholecystectomy. Surg Endosc 2015; 29:2837-2840.

28 Misrani JK, lqbal S, Sasoli NA, Memon ZA, Ahmedani SA. Comparative study of clinical profile in patients with solitary versus multiple gall stone. $J$ Liaquat Univ Med Health Sci 2016; 15:12-15.

29 Sperber AD, Dumitrascu D, Fukudo S, Gerson C, Ghoshal UC, Gwee KA, et al. The global prevalence of IBS in adults remains elusive due to the heterogeneity of studies: a Rome Foundation working team literature review. Gut 2017; 66:1075-1082.

30 Beckingham IJ. ABC of diseases of liver, pancreas, and biliary system. Gallstone disease. BMJ 2001; 322:91-94.

31 Lill S, Rantala A, Karvonen J, Polonen T, Gronroos JM. Elective laparoscopic cholecystectomy for symptomatic uncomplicated gallstone disease: do the symptoms disappear? Surg Endosc 2014; 28:1816-1820.

32 Lamberts MP, Den Oudsten BL, Gerritsen JJ, Roukema JA, Westert GP, Drenth JP, et al. Prospective multicentre cohort study of patient-reported outcomes after cholecystectomy for uncomplicated symptomatic cholecystolithiasis. Br J Surg 2015; 102:1402-1409.

33 Mjaland $\mathrm{OH}$, Høgevold HE, Buanes T. Standard preoperative assessment can improve outcome after cholecystectomy. Eur J Surg 2000; 166:129-135.

34 De Reuver PR, van Dijk AH, Wennmacker SZ, Lamberts MP, Boerma D, den Oudsten BL, et al. A randomized controlled trial to compare a restrictive strategy to usual care for the effectiveness of cholecystectomy in patients with symptomatic gallstones (SECURE trial protocol). BMC Surg 2016; 16:46.

35 Wennmacker SZ, Dijkgraaf MGW, Westert GP, Drenth JPH, van Laarhoven C, de Reuver PR. Persistent abdominal pain after laparoscopic cholecystectomy is associated with increased healthcare consumption and sick leave. Surgery 2018; 163:661-666.

36 Glambek I, Arnesjø B, Søreid O. Correlation between gallstones and abdominal symptoms in a random population: results from a screening study. Scand J Gastroenterol 2009; 24:277-281.

37 Corazziari E, Attili AF, Angeletti C, De Santis A. Gallstones, cholecystectomy and irritable bowel syndrome (IBS) MICOL populationbased study. Dig Liver Dis 2008; 40:944-950.

38 Kraag N, Thijs C, Knipschild P. Dyspepsia- how noisy are gallstones? A meta-analysis of epidemiologic studies of biliary pain, dyspeptic symptoms, and food intolerance. Scand J Gastroenterol 1995; 30:411-421.

39 Berger MY, van der Velden J, Lijmer JG, de Kort H, Prins A, Bohnen AM. Abdominal symptoms: do they predict gallstones? A systematic review. Scand J Gastroenterol 2000; 35:70-76.

40 Portincasa P, Moschetta A, Palasciano G. Cholesterol gallstone disease. Lancet 2006; 368:230-239.

41 Stroup DFB, Berlin JA, Morton SC, Olkin I, Williamson GD, Rennie D, et al. Meta-analysis of observational studies in epidemiology. JAMA 2000; 283:2008-2012.

42 Friedman GD. Natural history of asymptomatic and symptomatic gallstones. Am J Surg 1993; 165:399-404.

43 Mostafa R. Rome III: the functional gastrointestinal disorders, third edition, 2006. World J Gastroenterol 2008; 14:2124.

44 Pelitari S, Hathaway C, Gritton D, Smith A, Bush D, McKaig B. Impact and cost effectiveness of formal gastroenterology outpatient referral clinical assessment service. Gut 2017; 66 (Suppl 2):A8.

45 Feinle-Bisset C, Azpiroz F. Dietary and lifestyle factors in functional dyspepsia. Nat Rev Gastroenterol Hepatol 2013; 10:150-157. 